\title{
Alternative solutions to multi-variate control performance assessment problems is
}

\author{
Biao Huang ${ }^{\mathrm{a}, *}$, Steven X. Ding ${ }^{\mathrm{b}}$, Nina Thornhill ${ }^{\mathrm{c}}$ \\ a Department of Chemical and Materials Engineering, University of Alberta, Edmonton, AB, Canada T6G 2 G6 \\ ${ }^{\mathrm{b}}$ University of Duisburg-Essen, Institute for Automatic Control and Complex Systems/Faculty 5, \\ Bismarckstrasse 81, BB511, 47048 Duisburg, Germany \\ ${ }^{\mathrm{c}}$ Department of Electronic and Electrical Engineering, University College London, Torrington Place, WC1E 7JE London, UK
}

Received 2 December 2004; received in revised form 9 September 2005; accepted 9 September 2005

\begin{abstract}
Performance assessment of multi-variate control with minimum variance control as the benchmark requires an interactor matrix to filter the closed-loop output. This is to transfer the coordinate of the original variables into a new one in order to identify the control invariant disturbance dynamics from the first few terms of the closed-loop output Markov parameters. There has been a great deal of interest to simplify this approach, in particular, to find methods that do not need the interactor matrix. With this motivation, this paper explores alternative solutions to multi-variate control performance assessment problems. In particular, we will consider two practical scenarios: (1) known time delays between each pair of inputs and outputs, (2) no a priori knowledge about the process model or time delays at all. Solutions to these two scenarios are proposed. Two data-driven algorithms based on subspace approach are derived for the calculation of performance measures. Several examples illustrate the feasibility of the proposed approaches.
\end{abstract}

(C) 2005 Elsevier Ltd. All rights reserved.

Keywords: Performance monitoring; Performance assessment; Control monitoring; Multi-variate systems; Subspace methods; Projection

\section{Introduction}

The research on control loop performance monitoring and diagnostics has been and remains to be one of the most active research areas in process control over the last decade. It is estimated that several hundreds of papers have published in this or related direction [9]. On the practical side, Eastman Kodak recently reported regular loop monitoring on over 14,000 PID loops. Despite the success in the research and the applications of univariate control monitoring, applications of multi-variate control performance assessment remain as a challenge.

\footnotetext{
A short version of this paper was presented in 2004 IFAC DYCOPS

* Corresponding author. Tel.: +1 780 4929016; fax: +1 7804922881.

E-mail addresses: biao.huang@ualberta.ca (B. Huang), s.x.ding@ uni-duisburg.de (S.X. Ding), n.thornhill@ee.ucl.ac.uk (N. Thornhill).
}

Among a number of approaches for control performance monitoring, minimum variance control (MVC)benchmark remains the most popular benchmark. One of the reasons for the suitability of MVC benchmark to assess performance of control loops in the industry is that it is non-intrusive and routine closed-loop operating data are sufficient for the calculation of this benchmark $[6,26,12]$. However, this convenience holds only in the univariate case where the time delay is the only a priori knowledge that needs to be available. For multi-variate processes, this simplicity is lost and the time delay is no longer a simple technical concept. An interactor matrix is needed for multi-variate process, and its calculation is beyond the knowledge of the time delay between each pair of inputs and outputs. The earlier work in this area is Huang et al. $[13,14]$ and Harris et al. [7]. Both approaches require an explicit knowledge of the interactor matrix. 
In recent years, there are growing research interests in reducing the complexity of the a priori knowledge requirement, such as Ko and Edgar [18], Kadali and Huang [16], and $\mathrm{McNabb}$ and Qin [21]. Although these attempts have reduced the complexity of the a priori knowledge requirement to some extent, they all require certain information that is computationally simpler but fundamentally equivalent to the interactor matrices, for example, the open-loop process Markov parameter matrices, the lower triangular Toeplitz matrix, or the multi-variate time delay (MTD) matrix. That is, they all require a priori knowledge that is beyond the pure time delays between each pair of the inputs and outputs. Harris et al. [7,8] introduced an extended horizon performance monitoring approach without using the interactor matrix. Kadali and Huang [17] and Shah et al. [25] introduced curvature measures of multivariate performance without relying on the interactor matrix. Most recently, Huang et al. [11] proposed an algorithm for multi-variate control performance assessment with a priori knowledge of the order of the interactor matrix (OIM).

In the univariate case, one interprets output variance under minimum variance control as the variance of the optimal prediction error for the given time delay of a process. One can imagine that if a closed-loop output is highly predictable, then one should be able to do better, i.e. to compensate the predictable content by a well designed controller. Should a better controller be implemented, then the closed-loop output would have been less predictable. Therefore, the high predictability of a closed-loop output implies the potential to improve its performance by control re-tuning and/or re-design, or in other word, the existing controller may not have been satisfactory in terms of exploring its potential.

The actual process often has time delays, which prevent the complete compensation of the predictable content of the output. For example, if a univariate process has two sample time delays, then the compensation control action will not take effect on the output until two steps later and the one step ahead prediction will not be useful for its compensation. In this case, the best a controller can do is to compensate the predicted content according to the two-step optimal prediction (multi-step optimal prediction) and the minimum control error will coincide with the two-step prediction error. Therefore, the two-step optimal prediction error is the lower bound of the output error that can be achieved by a feedback controller. This lower bound is also known as the minimum variance that is often used for control loop performance assessment [6].

Although the same rationale cannot be exactly carried over to multi-variate processes due to the relatively complex delay structure for multi-variate processes, multi-step optimal predictions provide useful information about the control performance [23]. The multi-step optimal prediction error is analogous to closed-loop step response of the univariate process from the white noise to the output. The analogy provides an interesting interpretation of the multi-step optimal prediction error for multi-variate processes and results in a new measure for performance assessment.

While the prediction error based approach may be generic, if certain process knowledge such as time delays between each pair of inputs and outputs is available, one may be able to do more about control performance assessment than the prediction error based approach. It is known that the diagonal form of the interactor matrix only depends on the pair-wise time delays (delays between each pair of the inputs and outputs). If one can determine that the process has the diagonal form of the interactor, the computation for the performance assessment can be greatly simplified [12]. Thus there is a need to determine whether a process has a diagonal interactor matrix from the given pair-wise time delays.

Motivated by the above discussions, this paper is concerned with (1) development and analysis of an alternative performance assessment approach based on optimal predictions, (2) development of two data-driven algorithms to estimate the multi-step optimal prediction error variance, (3) methods to determine whether the process has a simple or diagonal form of interactor matrices. The remainder of this paper is organized as follows. In Section 2, the concept of the interactor matrix is revisited. The performance assessment problem, when the time delays between each pair of inputs and outputs are known, is discussed in Section 3. Results on multi-variate feedback control performance assessment without relying on any a priori knowledge of the process model are presented in Section 4. Data-driven algorithms for the computation of multi-step prediction error variance are derived in Section 5, followed by concluding remarks in Section 6.

\section{Revisit of interactor matrix}

Consider the following multi-variate process

$Y_{t}=T U_{t}+N a_{t}$

where $T$ and $N$ are proper (causal), rational transfer function matrices in the backshift operator $q^{-1} ; Y_{t}, U_{t}$ and $a_{t}$ are output, input and noise vectors of appropriate dimensions. $a_{t}$ is further assumed to be white noise with zero mean and $\operatorname{Var}\left(a_{t}\right)=\Sigma_{a} . N$ is rational realization of disturbance spectrum with the standard assumptions [20] that $N\left(q^{-1}=0\right)=I$ and $N$ is minimum phase, both of which are true through an appropriate realization of the disturbance spectrum.

Lemma 1. For every $n \times m$ proper, rational polynomial transfer function matrix $T$, there exists non-singular, $n \times n$ (non-unique) polynomial matrix $D$, such that $|D|=q^{r}$, $D^{\mathrm{T}} D=I$ and

$\lim _{q^{-1} \rightarrow 0} D T=\lim _{q^{-1} \rightarrow 0} \widetilde{T}=K$

where $K$ is a full rank constant matrix, the integer $r$ is defined as the number of infinite zeros of $T$, and $\widetilde{T}$ is the delay-free 
transfer function (factor) matrix of $T$ which contains only finite zeros. The matrix $D$ is known as the unitary interactor matrix, an equivalent form of the conventional lower triangular interactor matrix [3] and can be written as

$D=D_{0} q^{d}+D_{1} q^{d-1}+\cdots+D_{d-1} q$

where $d$ is denoted as the order of the interactor matrix and is unique for a given transfer function matrix $[5,24,22]$, and $D_{i}$ (for $i=0, \ldots, d-1)$ are coefficient matrices.

The interactor matrix $D$ can be one of the three forms described in the sequel. If $D$ is of the form: $D=q^{d} I$, then the transfer function matrix $T$ is regarded as having a simple interactor matrix. If $D$ is a diagonal matrix, i.e., $D=\operatorname{diag}\left(q^{d_{1}}, q^{d_{2}}, \ldots, q^{d_{n}}\right)$, then $T$ is regarded as having a diagonal interactor matrix. Otherwise, $T$ is considered to have a general interactor matrix.

The computation of the interactor matrix needs a complete process model or at least the first few Markov parameters of the process model [12], which is beyond the knowledge of time delays between each pair of the inputs and outputs. This requirement of process model information has been the main difficulty to the application of the multi-variate control performance assessment technique.

\section{Assessment of multi-variate control performance with known pair-wise time delays}

It has been shown in [12,7] that the first $d$ terms of the following moving average expansion of the interactor filtered multi-variate closed-loop output are feedback control invariant, where $d$ is the order of the interactor matrix.

$$
\begin{aligned}
\widetilde{Y}_{t} & =q^{-d} D Y_{t} \\
& =\widetilde{F}_{0} a_{t}+\widetilde{F}_{1} a_{t-1}+\cdots+\widetilde{F}_{d-1} a_{t-(d-1)}+\widetilde{F}_{d} a_{t-d}+\cdots
\end{aligned}
$$

The first $d$ terms represent the closed-loop output of $\widetilde{Y}_{t}$ if the minimum variance feedback control is implemented, where the minimum variance is in the sense of minimizing the trace of the covariance of $\widetilde{Y}$. Due to the property of the unitary interactor matrix, the trace of the covariance of $\widetilde{Y}_{t}$ is the same as that of $Y_{t}$. If the interactor matrix is known, then Eq. (3) can be easily obtained through time series analysis of $Y_{t}$ followed by the filtering of $q^{-d} D$ and then the moving average expansion, and the minimum variance term can be calculated, which can be used as a benchmark for multi-variable control performance assessment.

The problem in practical application is the interactor matrix as discussed in the last section, calculation of which, except for the diagonal interactor matrix, needs a priori knowledge of the process model. In particular, an experiment and identification effort has to be undertaken in order to calculate the interactor matrix.

Unlike univariate control performance assessment, for multi-variate control performance assessment, knowing pair-wise time delays is not sufficient for calculating minimum variance unless the interactor matrix has a simple or diagonal structure. However, if the time delays between each pair of inputs and outputs are indeed known, we should search for a possible simple or diagonal structure of the interactor matrix, which can directly lead to the computation of the multi-variate minimum variance. Both the simple and the diagonal interactor matrices can be calculated from the time delays between each pair of inputs and outputs of the process. One may surprisingly find that the simple and diagonal interactor matrices are not uncommon, particularly in industrial process, where the sparse structure of the transfer function matrix is often observed. The sparse structure also facilitates the determination of the interactor structure.

Consider a multi-variable transfer function matrix of dimension $n \times m$ given by

$$
T=\left(\begin{array}{cccc}
T_{11} q^{-d_{11}} & T_{12} q^{-d_{12}} & \ldots & T_{1 m} q^{-d_{1 m}} \\
T_{21} q^{-d_{21}} & T_{22} q^{-d_{22}} & \ldots & T_{2 m} q^{-d_{2 m}} \\
\ldots & \ldots & \ldots & \ldots \\
T_{n 1} q^{-d_{n 1}} & T_{n 2} q^{-d_{n 2}} & \ldots & T_{n m} q^{-d_{n m}}
\end{array}\right)
$$

where $T_{i j}$ is a scalar transfer function from the $j$ th input to the $i$ th output. Define a delay matrix

$$
\Psi=\left(\begin{array}{cccc}
t_{11} q^{-d_{11}} & t_{12} q^{-d_{12}} & \cdots & t_{1 m} q^{-d_{1 m}} \\
t_{21} q^{-d_{21}} & t_{22} q^{-d_{22}} & \cdots & t_{2 m} q^{-d_{2 m}} \\
\cdots & \cdots & \cdots & \cdots \\
t_{n 1} q^{-d_{n 1}} & t_{n 2} q^{-d_{n 2}} & \cdots & t_{n m} q^{-d_{n m}}
\end{array}\right)
$$

where $d_{i j}$ 's are time delays that are assumed known; $t_{i j}$ is the first non-zero impulse response coefficient from the $j$ th input to the $i$ th output, which is typically unknown. From $\Psi$, we can obtain a diagonal matrix

$$
\Theta=\left(\begin{array}{cccc}
q^{d_{1}} & & & \\
& q^{d_{2}} & & \\
& & \ddots & \\
& & & q^{d_{n}}
\end{array}\right)
$$

where $d_{i}=\min \left\{d_{i j}: j=1, \ldots, m\right\}$. Then the following lemma is true:

Lemma 2. If $T$ has a diagonal interactor matrix $D$ then $D=\Theta$, where $T$ and $\Theta$ are given by Eqs. (4) and (6), respectively.

Proof. Use the method of contradiction.

If $T$ has a diagonal interactor matrix $D$, but $D \neq \Theta$. This means there exists at least one diagonal element in $D$, say, the $i$ th diagonal element, such that

$$
D=\left(\begin{array}{ccccc}
q^{d_{1}} & & & & \\
& \ddots & & & \\
& & q^{+l} & & \\
& & & \ddots & \\
& & & & q^{+d_{n}}
\end{array}\right)
$$


where $q^{-l}$ is the $i$ th diagonal element and $l>d_{i}$. Multiplying $T$ by $D$ yields

$$
D T=\left(\begin{array}{ccccc}
T_{11} q^{-\left(d_{11}-d_{1}\right)} & \ldots & \ldots & \ldots & T_{1 m} q^{-\left(d_{1 m}-d_{1}\right)} \\
\ldots & \ldots & \ldots & \ldots & \ldots \\
T_{i 1} q^{-\left(d_{i 1}-l\right)} & \ldots & T_{i p} q^{-\left(d_{i p}-l\right)} & \ldots & T_{i m} q^{-\left(d_{i m}-l\right)} \\
\ldots & \ldots & \ldots & \ldots & \ldots \\
T_{n 1} q^{-\left(d_{n 1}-d_{n}\right)} & \ldots & \ldots & \ldots & T_{n m} q^{-\left(d_{n m}-d_{n}\right)}
\end{array}\right)
$$

Since $l$ is not the minimum of $\left\{d_{i j}: j=1, \ldots, m\right\}$, there exists at least one element in the $i$ th row, say at the $p$ th column, such that $-\left(d_{i p}-l\right)>0$. Therefore $\lim _{q^{-1} \rightarrow 0} q^{-\left(d_{i p}-l\right)} \rightarrow \infty$ and $\lim _{q^{-1} \rightarrow 0} D T$ does not exist. Consequently $D$ cannot be an interactor matrix of $T$.

The following lemma provides a useful criterion to determine whether a process $T$ has a diagonal interactor matrix:

Lemma 3. If $K=\lim _{q^{-1} \rightarrow 0} \Theta \Psi$ is of full rank for all $t_{i j} \neq 0$, then (1) the interactor matrix is diagonal and (2) $D=\Theta$.

Proof. The first part can be proved by noticing the fact that $\lim _{q^{-1} \rightarrow 0} \Theta T=\lim _{q^{-1} \rightarrow 0} \Theta \Psi=K$. By the definition of the interactor matrix, if $K$ is of full rank, $\Theta$ must be an interactor matrix of $T$. Based on the result of the first part, the second part of the proof directly follows from Lemma 2 .

Lemma 3 provides a sufficient condition for the determination of the interactor structure. In practice, one can relax this condition by checking if the determinant of $K$ is zero (if $K$ is not a square matrix, one instead has to use $K^{\mathrm{T}} K$ or $K K^{\mathrm{T}}$ for tall and fat $K$ respectively), to determine conditions for the singularity. MATLAB symbolic toolbox is useful for such an application. The procedure is as follows: (1) calculate the determinant, (2) find condition for the determinant to be zero, and (3) check whether these conditions hold.

Next, we will demonstrate the method of determining the structure of the interactor matrix using several classical multi-variable examples and an industrial example.

\subsection{Examples}

Example 4. Consider four $2 \times 2$ processes discussed in [15]. The transfer functions matrices are given in Table 1.

With sampling interval $T_{\mathrm{s}}=1$, the four continuous-time transfer function matrices can be transferred to discrete-time transfer function matrices (by assuming zero-order hold). The time delay matrices $\Psi$ are summarized in the first row of Table 2. The $\Theta$ matrices are obtained and summarized in the second row. The multiplications $K=\lim _{q^{-1} \rightarrow 0} \Theta \Psi$ are listed in the third row, and their determinants are shown in the fourth row. The fifth row shows the conditions for the determinants to be zero. It is not difficult to find out that Wood-Berry and Wardle-Wood both have the diagonal interact matrices; Ogunnaike and Ray has the simple interactor matrix structure unless the first non-zero impulse responses of the four sub-transfer functions satisfy the condition $\frac{t_{11}}{t_{21}}=\frac{t_{12}}{t_{22}}$, which is not the case; Vinante-Luyben does not have the simple or diagonal interactor matrix.

Example 5. Consider an industrial $6 \times 6$ process presented in [4], which has the following pair-wise delay matrix:

$$
\Psi=\left(\begin{array}{cccccc}
q^{-1} & 0 & 0 & 0 & 0 & 0 \\
q^{-1} & q^{-1} & q^{-1} & 0 & q^{-1} & 0 \\
q^{-1} & q^{-1} & q^{-1} & 0 & q^{-1} & 0 \\
0 & 0 & 0 & 0 & q^{-1} & 0 \\
q^{-2} & q^{-2} & 0 & q^{-2} & q^{-2} & q^{-2} \\
0 & 0 & 0 & q^{-1} & q^{-1} & 0
\end{array}\right)
$$

An unitary interactor matrix was calculated in [4] using complete knowledge of the process transfer function matrix. The result was

$$
D=\left(\begin{array}{cccccc}
0.05925 q & 0.7234 q & 0.4 q & -0.5596 & 0 & 0 \\
0.006454 q & 0.4251 q & -0.9003 q & -0.0934 q & 0 & 0 \\
-0.02356 q & -0.3103 q & -0.09755 q & -0.4734 q & 0 & 0.8183 q \\
-0.03354 q & -0.4417 q & -0.1389 q & -0.6739 q & 0 & -0.5748 q \\
-0.9974 q & 0.06791 q & 0.02491 q & 0 & 0 & 0 \\
0 & 0 & 0 & 0 & q^{2} & 0
\end{array}\right)
$$

\begin{tabular}{|c|c|c|c|c|}
\hline Process & $g_{11}(s)$ & $g_{12}(s)$ & $g_{21}(s)$ & $g_{22}(s)$ \\
\hline \multirow[t]{2}{*}{ Wood and Berry (WB) } & $12.8 e^{-s}$ & $-18.9 e^{-3 s}$ & $6.6 e^{-7 s}$ & $-19.4 e^{-3 s}$ \\
\hline & $\overline{16.7 s+1}$ & $\overline{21 s+1}$ & $\overline{10.9 s+1}$ & $\overline{14.4 s+1}$ \\
\hline \multirow{2}{*}{ Vinante and Luyben (VL) } & $-2.2 e^{-s}$ & $1.3 e^{-0.3 s}$ & $-2.8 e^{-1.8 s}$ & $4.3 e^{-0.35 s}$ \\
\hline & $\overline{7 s+1}$ & $7 s+1$ & $9.5 s+1$ & $\overline{9.2 s+1}$ \\
\hline \multirow{2}{*}{ Wardle and Wood (WW) } & $0.126 e^{-6 s}$ & $-0.101 e^{-12 s}$ & $0.094 e^{-8 s}$ & $-0.12 e^{-8 s}$ \\
\hline & $\overline{60 s+1}$ & $\overline{(48 s+1)(45 s+1)}$ & $38 s+1$ & $\overline{35 s+1}$ \\
\hline \multirow{2}{*}{ Ogunnaike and Ray (OR) } & $22.89 e^{-0.2 s}$ & $-11.64 e^{-0.4 s}$ & $4.689 e^{-0.2 s}$ & $5.8 e^{-0.4 s}$ \\
\hline & $\overline{4.572 s+1}$ & $1.807 s+1$ & $\overline{2.174 s+1}$ & $\overline{1.801 s+1}$ \\
\hline
\end{tabular}

Table 1

Four classical multi-variable processes 
Table 2

Determination of interactor structure for four classical multivariable processes

\begin{tabular}{|c|c|c|c|c|}
\hline & WB & VL & WW & OR \\
\hline $\bar{\Psi}$ & $\left(\begin{array}{ll}t_{11} q^{-2} & t_{12} q^{-4} \\
t_{21} q^{-8} & t_{22} q^{-4}\end{array}\right)$ & $\left(\begin{array}{ll}t_{11} q^{-2} & t_{12} q^{-1} \\
t_{21} q^{-2} & t_{22} q^{-1}\end{array}\right)$ & $\left(\begin{array}{cc}t_{11} q^{-7} & t_{12} q^{-14} \\
t_{21} q^{-9} & t_{22} q^{-9}\end{array}\right)$ & $\left(\begin{array}{ll}t_{11} q^{-1} & t_{12} q^{-1} \\
t_{21} q^{-1} & t_{22} q^{-1}\end{array}\right)$ \\
\hline$\Theta$ & $\left(\begin{array}{ll}q^{2} & \\
& q^{4}\end{array}\right)$ & $\left(\begin{array}{ll}q & \\
& q\end{array}\right)$ & $\left(\begin{array}{ll}q^{7} & \\
& q^{9}\end{array}\right)$ & $\left(\begin{array}{ll}q & \\
& q\end{array}\right)$ \\
\hline$K$ & $\left(\begin{array}{cc}t_{11} & 0 \\
0 & t_{22}\end{array}\right)$ & $\left(\begin{array}{ll}0 & t_{12} \\
0 & t_{22}\end{array}\right)$ & $\left(\begin{array}{cc}t_{11} & 0 \\
t_{21} & t_{22}\end{array}\right)$ & $\left(\begin{array}{ll}t_{11} & t_{12} \\
t_{21} & t_{22}\end{array}\right)$ \\
\hline
\end{tabular}

Using the method discussed in this section, without knowing process models other than the delays we would get

$$
\Theta=\left(\begin{array}{cccccc}
q & & & & & \\
& q & & & & \\
& & q & & & \\
& & q & & \\
& & & q^{2} & \\
& & & & q
\end{array}\right)
$$

and $K$ matrix

$$
K=\left(\begin{array}{cccccc}
t_{11} & 0 & 0 & 0 & 0 & 0 \\
t_{21} & t_{22} & t_{23} & 0 & t_{25} & 0 \\
t_{31} & t_{32} & t_{33} & 0 & t_{35} & 0 \\
0 & 0 & 0 & 0 & t_{45} & 0 \\
t_{51} & t_{52} & 0 & t_{54} & t_{55} & t_{56} \\
0 & 0 & 0 & t_{64} & t_{65} & 0
\end{array}\right)
$$

The determinant of $K$ can be calculated as $\operatorname{det}(K)=$ $t_{25} t_{64} t_{56}\left(t_{22} t_{33}-t_{32} t_{23}\right)$. Due to the sparse structure of industrial processes, the determinants often have such a simple structure. Since $t_{i j} \neq 0$ by the definition, the condition for the determinant to be zero is $\frac{t_{22}}{t_{32}}=\frac{t_{23}}{t_{33}}$. Whether this condition holds can be easily determined by checking the variables of the process. As discussed in [4], $C V_{2}$ is temperature, $C V_{3}$ internal reflux ratio, $M V_{2}$ reboiler stem flow, and $M V_{3}$ internal reflux flow. The chance for the condition to be true has the probability $\rightarrow 0$. Therefore, the interactor matrix should have a diagonal structure and the complete knowledge of the multi-variate process, which was assumed in [4], is not necessary. In the worst case, if one is not able to determine whether the condition is true, one would at most need to find out the first non-zero impulse response coefficients of four sub-transfer functions, a significant reduction of the a priori knowledge than the complete transfer function matrices.

Is this result in a contradiction to that of [4] shown in Eq. (10)? It has been shown in [12] that the two unitary interactor matrices are equivalent if

$\bar{D}=\Gamma D$

where $\Gamma$ is a unitary constant matrix. It can be shown by using QR decomposition that if

$$
\Gamma=\left(\begin{array}{cccccc}
-0.0592 & -0.0065 & 0.0236 & 0.0330 & 0.9974 & 0 \\
-0.7234 & -0.4251 & 0.3103 & 0.4417 & -0.0679 & 0 \\
0.4000 & -0.9003 & -0.0976 & -0.1389 & 0.0249 & 0 \\
-0.5596 & -0.0934 & -0.4734 & -0.6738 & 0 & 0 \\
0 & 0 & 0 & 0 & 0 & 1 \\
0 & 0 & 0.8183 & -0.5748 & 0 & 0
\end{array}\right)
$$

Pre-multiplying Eq. (10) by $\Gamma$ results in Eq. (11), a diagonal interactor matrix! Therefore, although it is not straightforward to see it, Eq. (10) is in fact a diagonal interactor matrix.

Once the diagonal structure of the interactor matrix is determined, the multi-variate minimum variance can be determined by following the approaches discussed in $[7,12]$, and is omitted here.

\section{Assessment of multi-variate control performance without any a priori knowledge of interactor matrices}

If the pair-wise time delays are unknown or the interactor matrix has been determined to be non-diagonal, then it is not possible to estimate minimum variance from closedloop routine operating data. In this section, we shall consider an alternative method for the assessment of multi-variate control loop performance without relying on any a priori knowledge of the interactor matrices. There are several interactor matrix-free methods in the literature, mainly based on closed-loop impulse response [12,23,25], and variance of multi-step prediction errors [7,17,23]. Earlier work in using interactor-free approach may be traced back to $[1,2]$. In this section, we shall extend the above mentioned methods, mainly the variance of prediction error based methods, to a novel closed-loop potential graphic measure and a single numerical value to measure control performance potential.

Consider a closed-loop multi-variate process represented by a moving average or a Markov parameter form:

$Y_{t}=F_{0} a_{t}+F_{1} a_{t-1}+\cdots+F_{i-1} a_{t-(i-1)}+F_{i} a_{t-i}+\cdots$

We have assumed $\Sigma_{a}=I$. However, if $\Sigma_{a} \neq I$, one can always normalize $F_{i}$ 's such that $F_{i} \leftarrow F_{i} \Sigma_{a}^{1 / 2}$ and $a_{t} \leftarrow \Sigma_{a}^{-1 / 2} a_{t}$, and then the new $a_{t}$ will satisfy $\Sigma_{a}=I$. This 
moving average model can be estimated from routine operating data without any a priori knowledge about the interactor matrices.

Since $a_{t}$ is white noise, the optimal $i$ th step prediction is given by

$Y_{t \mid t-i}=F_{i} a_{t-i}+F_{i+1} a_{t-i-1}+\cdots$

and the prediction error $e_{t \mid t-i}=Y_{t}-Y_{t \mid t-i}$ is given by

$e_{t \mid t-i}=F_{0} a_{t}+F_{1} a_{t-1}+\cdots+F_{i-1} a_{t-(i-1)}$

The covariance of the prediction error can be calculated as

$\operatorname{Cov}\left(e_{t \mid t-i}\right)=F_{0} F_{0}^{\mathrm{T}}+F_{1} F_{1}^{\mathrm{T}}+\cdots+F_{i-1} F_{i-1}^{\mathrm{T}}$

and its scalar measure

$s_{i} \triangleq \operatorname{tr}\left[\operatorname{Cov}\left(e_{t \mid t-i}\right)\right]=\operatorname{tr}\left(F_{0} F_{0}^{\mathrm{T}}+F_{1} F_{1}^{\mathrm{T}}+\cdots+F_{i-1} F_{i-1}^{\mathrm{T}}\right)$

The incremental of the prediction error can be calculated as $r_{i} \triangleq \operatorname{tr}\left[\operatorname{Cov}\left(e_{t \mid t-i}\right)-\operatorname{Cov}\left(e_{t \mid t-(i-1)}\right)\right]=\operatorname{tr}\left(F_{i-1} F_{i-1}^{\mathrm{T}}\right)$

If we plot $s_{i}$ versus $i$, then the plot reflects how the prediction error increases with the prediction horizon. Note that as $i \rightarrow \infty, \operatorname{Cov}\left(e_{t \mid t-i}\right) \rightarrow \operatorname{Cov}\left(Y_{t}\right)$. This fact can be seen by comparing Eqs. (15) and (17).

Remark 6. $s_{i}$ is nothing but the sum of squared error of the closed-loop response to an impulse disturbance up to time $i$ if the disturbance is an impulse. If we plot $s_{i}$ versus $i$, each point of the plot, for example $s_{i}$, represents the sum of squared error (SSE) of the closed-loop response up to time $i$. If, after time $i$, the disturbance can be completely controlled (by a deadbeat control for example), then the total error of the response will remain to be $s_{i}$, which is the $i$ step optimal prediction error from stochastic view point. Since $s_{i}$ is the integration of the squared closed-loop impulse response, it is analogous to the step response and can be used to determine dynamic information such as the settling time of the closed-loop response to the disturbance. As explained in [25], $r_{i}$ is a 2-norm measure of the impulse response coefficients and is analogous to the squared impulse response coefficients of a univariate process. Therefore, this plot of $r_{i}$ versus $i$, is also an indication of closed-loop performance of a multi-variate controller, which has been used in some commercial software for multi-variate control performance monitoring and also in the literature [12,25]. In [23], the idea of impulse response as a measure of control performance has been extended to individual impulse response of each output to each shock of the disturbances to measure the interaction of variables. They have also proposed the use of forecast error variance decomposition (FEVD) for the measure of interactions. While $s_{i}$ is the overall measure of prediction error variance, the FEVD is the decomposition of prediction error variance of individual variable to each shock of the disturbances.

Motivated by the interpretation of $r_{i}$ and $s_{i}$, we define the closed-loop potential $p_{i}$ as

$p_{i} \triangleq \frac{s_{\infty}-s_{i}}{s_{\infty}}$

Since $s_{i}$ is monotonically increasing with $i, p_{i}$ is monotonically decreasing. Since $s_{0}=\operatorname{tr}\left[\operatorname{Cov}\left(Y_{t}-Y_{t \mid t}\right)\right]=0, p_{0}=1$. Therefore, $p_{i}$ starts from 1 at $i=0$ and monotonically decreases to 0 and $0 \leqslant p_{i} \leqslant 1$. Unlike the impulse response or variance of prediction error, $p_{i}$ is dimensionless and facilitate the comparison of control performance. $p_{i}$ can be interpreted as follows: If a deadbeat control action can be applied from time $i$, then the process output SSE can be reduced by $100 \times p_{i}$ percent. From stochastic view point, if $i$ is greater than the interactor order $d$, it is possible that the variance of the multi-variate output can be reduced by $100 \times p_{i}$ percent of the current variance $[10,11]$. Since the order of the actual interactor matrix may not be known, one would look for the trajectory of the closed-loop potential versus a range of possible $d$. Potential plots such as those illustrated in Fig. 1 are useful. Faster decays of the potential to zero indicate less possibility to improve the control. Due to the monotonically decreasing nature of the potentials and fixed starting and ending values of the potentials, the area below the potential plot well reflects the rate of its decaying. Therefore, it is possible to define a scalar index to monitor the change of the closed-loop potential. This index is called relative closed-loop potential index and can be calculated as

$\eta_{p}=\frac{\sum p_{i}^{(2)}}{\sum p_{i}^{(1)}}-1$

where $p_{i}^{(1)}$ is a reference potential calculated, for example, from the data sampled before control tuning, and $p_{i}^{(2)}$

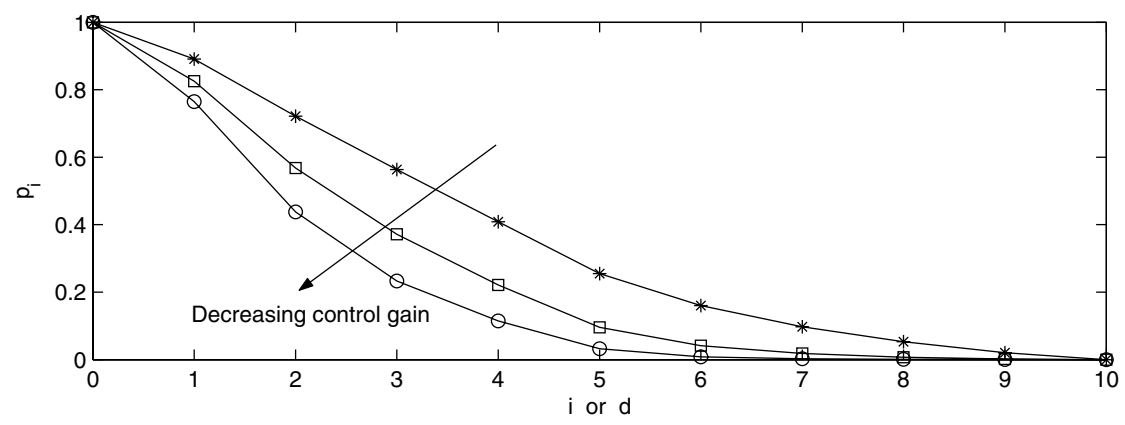

Fig. 1. Illustration of closed-loop potential $p_{i}$ plot. 
is calculated from data sampled after the tuning. The value of $\eta_{p}$ gives the percent change of the closed-loop potential with the positive sign indicating an increased potential and the negative sign indicating a decreased potential. Note that an increase of the potential implies a deteriorated tuning while a decrease of the potential implies an improved tuning. In analogy to FEVD [23], closed-loop potentials can also be defined for the individual output variable and the relative closed-loop potential index for each output can also be derived. To calculate potential of individual variable, the trace operator $\operatorname{tr}[\cdot]$ should be replaced by diagonalization operator $\operatorname{diag}[\cdot]$.

The closed-loop potential is an extension of variance of the prediction error. It is naturally related to all variance of prediction error based measures for performance assessment, such as $\mathrm{PI}_{3}(k)$ in [7] where only a fixed $k$ was considered though. However, this graphic extension, scalar measure of closed-loop potential, and its interpretations have provided enhancements to previously proposed methods.

\subsection{Example}

Example 7. Consider a $2 \times 2$ multi-variable process with the open-loop transfer function matrix $T$ and disturbance transfer function matrix $N$ given by

$$
T=\left[\begin{array}{cc}
\frac{q^{-1}}{1-0.4 q^{-1}} & \frac{0.5 q^{-2}}{1-0.1 q^{-1}} \\
\frac{0.3 q^{-1}}{1-0.4 q^{-1}} & \frac{q^{-2}}{1-0.8 q^{-1}}
\end{array}\right]
$$

$$
N=\left[\begin{array}{cc}
\frac{1}{1-0.5 q^{-1}} & \frac{-q^{-1}}{1-0.6 q^{-1}} \\
\frac{q^{-1}}{1-0.7 q^{-1}} & \frac{1.0}{1-0.8 q^{-1}}
\end{array}\right]
$$

The white noise excitation, $a_{t}$, is a two-dimensional normally distributed white noise sequence with $\Sigma_{a}=I$.

Consider that the following multi-loop controller is implemented in the process:

$$
Q=\left[\begin{array}{cc}
k \frac{0.5-0.20 q^{-1}}{1-0.5 q^{-1}} & 0 \\
0 & \frac{0.25-0.200 q^{-1}}{\left(1-0.5 q^{-1}\right)\left(1+0.5 q^{-1}\right)}
\end{array}\right]
$$

In this example, three controller gains, $k=2.8,2.9,3.0$ respectively, are considered. $s_{i}$ and $r_{i}$ for $i=1,2, \ldots, 10$ are calculated and plotted in Fig. 2. The $s_{i}$ plot (top panel) indicates that the closed-loop settling time increases with the increasing of the controller gain, so does the SSE. For example, the settling time for $k=2.8$ is about five samples while the settling time for $k=3.0$ is more than 10 samples. The $r_{i}$ plot shown in the bottom panel of Fig. 2 presents the similar information as $s_{i}$ such as the information about the settling time. However, unlike the $s_{i}$ plot which is monotonically increasing, the $r_{i}$ plot has a more complicated and hard-to-interpret pattern. We therefore recommend the use of the $s_{i}$ plot and the $p_{i}$ plot (to be discussed next). The $s_{i}$ plot or its equivalent has been discussed in [17,23].

The potential plot of $p_{i}$ shown in Fig. 3 is possibly more useful in the interpretation of control performance. For
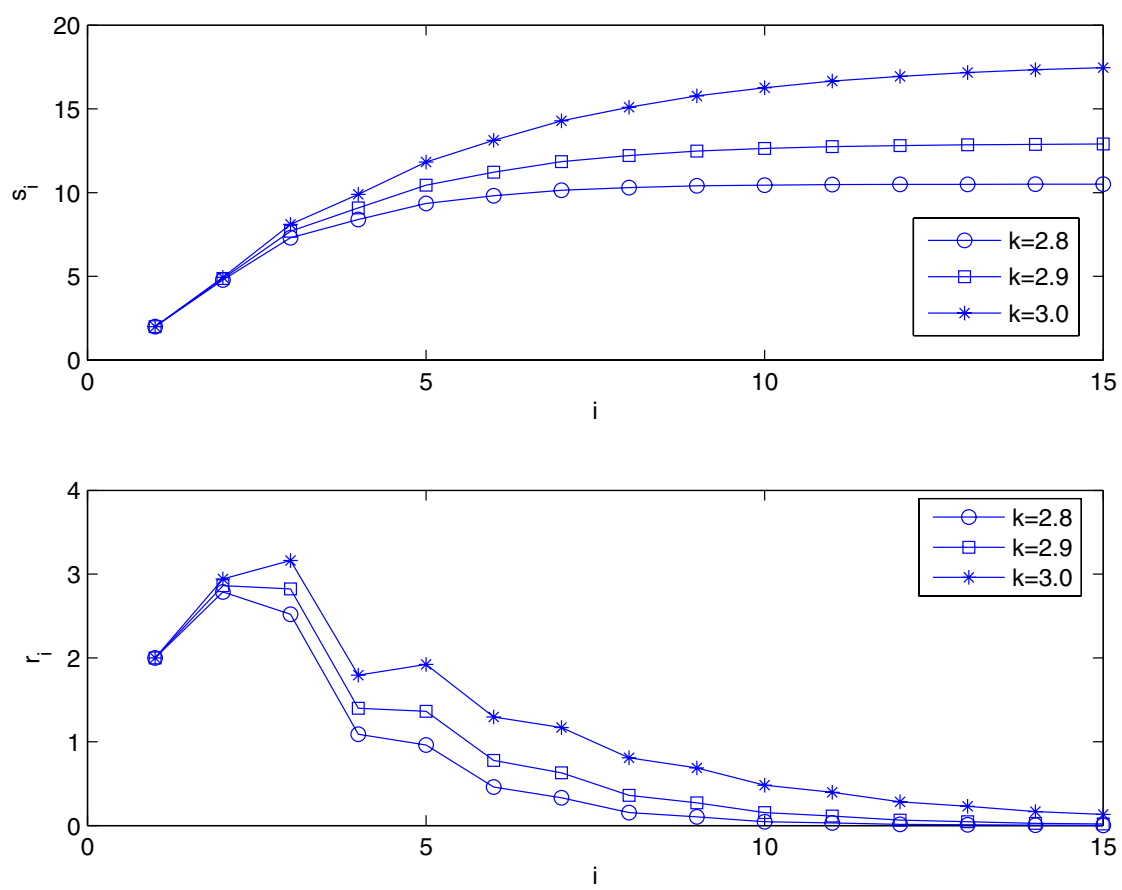

Fig. 2. $s_{i}$ and $r_{i}$ plots. 


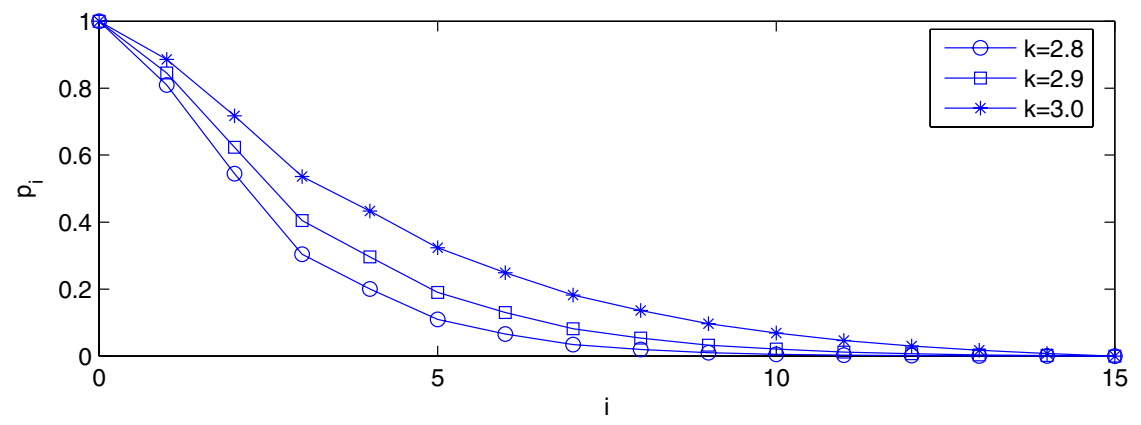

Fig. 3. $p_{i}$ plot.

example, $p_{i}$ for $k=3.0$ has a slowest rate to approach its steady state and thus its potential decreases to zero at the slowest rate. For a considerable range of the process delays (expressed by interactor order $d$ for example), its potential is significantly different from zero. As an example, for an interactor order up to 5 samples, the potential is larger than 0.3 , i.e. $30 \%$ reduction of variance is possible for the interactor order up to 5 . On the other hand, for the tuning of $k=2.8$, the potential dies to zero quickly. In this case, there is not much potential left after the interactor order is greater than 5 .

For control tuning of multi-variate systems or control upgrading from multi-loop control to multi-variable control such as MPC, one is interested in whether control performance is indeed improved. If an existing 1st controller gain is $k=2.9$, assume that the gain is tuned to 2.8 or 3.0 and representative closed-loop data are sampled before and after the tuning. Then the scalar measures of the relative closed-loop potentials calculated from the data are -0.16 or 0.28 for tunings $k=2.8$ or 3.0 with $k=3.0$ as the reference. These results indicate that (1) if the controller gain increases to 3.0 , then the resulting system has increased closed-loop potential by $28 \%$, indicating a deteriorated performance; (2) if the controller gain decreases to 2.8 , then the resulting system has reduced closed-loop potential by $16 \%$, indicating an improved performance.

The scalar measures of the individual relative closedloop potentials for the first output calculated from the data are -0.18 and 0.27 for tunings $k=2.8$ and 3.0, respectively. The scalar measures of the individual relative closed-loop potentials for the second output calculated from the data are -0.04 and 0.10 for tunings $k=2.8$ and 3.0 , respectively. These results indicate that the proposed tuning has much less effect on the second output than the first output. This fact can also be visualized from Fig. 4. More interesting elaboration of individual potentials will be discussed in a case study example shortly.
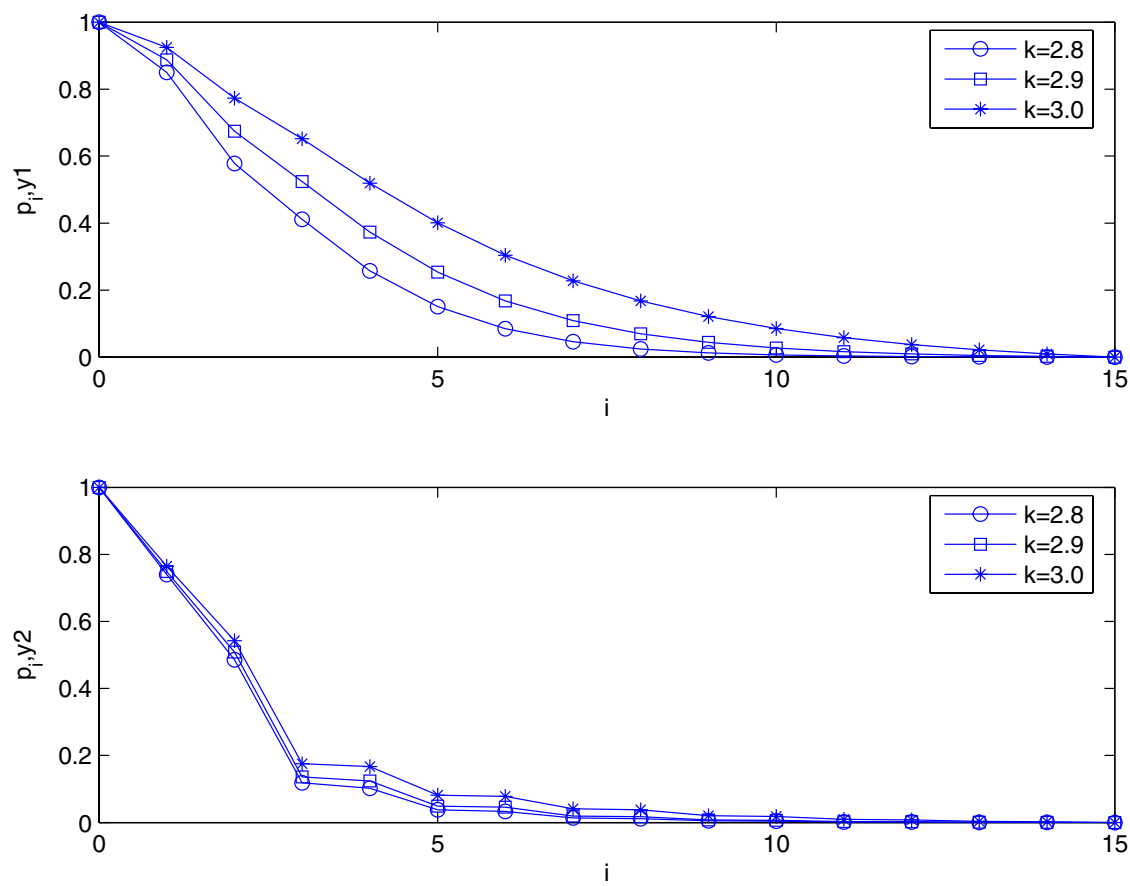

Fig. 4. Individual closed-loop potential. The top panel is closed-loop potential of $y_{1}$; the bottom panel is closed-loop potential of $y_{2}$. 


\section{Data-driven subspace approach to the calculation of multi-step optimal prediction errors}

\subsection{Preliminary_-subspace modeling}

Consider a time series model in innovation form

$x_{t+1}=A x_{t}+K a_{t}$

$Y_{t}=C x_{t}+a_{t}$

Note that, if one wishes, this parametric state space model (or equivalently ARMA model) can also be estimated from closed-loop routine operating data using a standard time series analysis software package. The closed-loop Markov parameter matrices can then be derived from the model, and finally prediction errors can be calculated. The procedure is, however, non-trivial for a multi-variate process. The challenge of using conventional time series analysis for multi-variate modeling lies in the following aspects:

- Difficulty in selection of model order [23]. Take a twovariable vector ARMA model as an example, $A Y=C e$ where $A$ is a $2 \times 2$ polynomial matrix. Each element of the matrix can have different order. Thus one may have to choose orders for four polynomials in matrix $A$. Similarly, $C$ has the same dimension as $A$ and could add order determination for additional four polynomials.

- Large number of parameters to be estimated. Suppose we choose each polynomial of $A$ and $C$ to be second order. Each second order polynomial has two parameters. Then there are potentially 16 parameters to estimate. This number grows quickly with the dimension of the output to be handled.

- Complexity of algorithms. In general, the ARMA model has to be estimated by a non-linear numerical optimization. The associated problems include the global versus local optimization, convergence, and computation load.

- The simpler AR model may be used to model the time series as well; however, it typically needs higher order than that of the ARMA model. Therefore it has even larger number of parameters to estimate. The difficulty and uncertainty in selecting the order of a vector AR model have also been discussed in [23].

Next, we will present two data-driven algorithms to calculate the multi-step optimal prediction errors, from which both $s_{i}$ and $p_{i}$ can easily be calculated without needing a model.

The relation between the state space representation and the moving average form (cf. Eq. (15)) can be easily established as the following:

$$
\begin{aligned}
& F_{0}=I \\
& F_{1}=C K \\
& F_{2}=C A K \\
& \vdots \\
& F_{i-1}=C A^{i-2} K
\end{aligned}
$$

Following the standard subspace notation [27], one can derive, through the iterative substitution of Eqs. (20) and (21), the subspace matrix equations as

$$
\begin{aligned}
& Y_{i \mid 2 i-1}=\Gamma_{i} X_{i}+H_{i}^{s} E_{i \mid 2 i-1} \\
& Y_{0 \mid i-1}=\Gamma_{i} X_{0}+H_{i}^{s} E_{0 \mid i-1} \\
& X_{i}=A^{i} X_{0}+\Delta_{i}^{s} E_{0 \mid i-1}
\end{aligned}
$$

where the output and innovation block-Hankel matrices are defined as

$$
\begin{aligned}
Y_{0 \mid i-1} & =\left(\begin{array}{cccc}
Y_{0} & Y_{1} & \cdots & Y_{j-1} \\
Y_{1} & Y_{2} & \cdots & Y_{j} \\
\cdots & \cdots & \cdots & \cdots \\
Y_{i-1} & Y_{i} & \cdots & Y_{i+j-2}
\end{array}\right) \\
E_{0 \mid i-1} & =\left(\begin{array}{cccc}
a_{0} & a_{1} & \cdots & a_{j-1} \\
a_{1} & a_{2} & \cdots & a_{j} \\
\cdots & \cdots & \cdots & \cdots \\
a_{i-1} & a_{i} & \cdots & a_{i+j-2}
\end{array}\right)
\end{aligned}
$$

Other output $Y_{i \mid 2 i-1}$ and innovation $E_{i \mid 2 i-1}$ block-Hankel matrices are defined conformably with the subscript being changed accordingly. The dimensions of these matrices have been discussed in [27].

The state is defined as

$$
\begin{aligned}
& X_{0}=\left(\begin{array}{llll}
x_{0} & x_{1} & \cdots & x_{j-1}
\end{array}\right) \\
& X_{i}=\left(\begin{array}{llll}
x_{i} & x_{i+1} & \cdots & x_{i+j-1}
\end{array}\right)
\end{aligned}
$$

The extended observability matrix $\Gamma_{i}$ is given as

$\Gamma_{i}=\left(\begin{array}{c}C \\ C A \\ C A^{2} \\ \cdots \\ C A^{i-1}\end{array}\right)$

The reversed extended controllability matrix $\Delta_{i}^{s}$ is given below

$$
\Delta_{i}^{s}=\left(\begin{array}{lllll}
A^{i-1} K & A^{i-2} K & \cdots & A K & K
\end{array}\right)
$$

The lower triangular block-Toeplitz matrix $H_{i}^{s}$ is given by

$$
H_{i}^{s}=\left(\begin{array}{ccccc}
I & 0 & 0 & \cdots & 0 \\
C K & I & 0 & \cdots & 0 \\
C A K & C K & I & \cdots & 0 \\
\cdots & \cdots & \cdots & \cdots & \cdots \\
C A^{i-2} K & C A^{i-3} K & C A^{i-4} K & \cdots & I
\end{array}\right)
$$

In subspace identification literature, the following shorthand notations are often used:

$$
\begin{aligned}
& Y_{p}=Y_{0 \mid i-1} \quad Y_{f}=Y_{i \mid 2 i-1} \\
& E_{p}=U_{0 \mid i-1} \quad E_{f}=U_{i \mid 2 i-1} \\
& X_{p}=X_{0} \quad X_{f}=X_{i}
\end{aligned}
$$


where subscript $p$ stands for the "past" and $f$ for the "future".

With these short-hand notations, the subspace Eqs. (23)-(25) can be written as

$Y_{f}=\Gamma_{i} X_{f}+H_{i}^{s} E_{f}$

$Y_{p}=\Gamma_{i} X_{p}+H_{i}^{s} E_{p}$

$X_{f}=A^{i} X_{p}+\Delta_{i}^{s} E_{p}$

\subsection{Calculation of multi-step optimal prediction errors}

Re-write Eq. (27) as

$Y_{f}-\Gamma_{i} X_{f}=H_{i}^{s} E_{f}$

We shall now evaluate the following quantity

$$
\begin{gathered}
\lim _{j \rightarrow \infty} \frac{1}{j}\left(Y_{f}-\Gamma_{i} X_{f}\right)\left(Y_{f}-\Gamma_{i} X_{f}\right)^{\mathrm{T}} \\
=\lim _{j \rightarrow \infty} \frac{1}{j} H_{i}^{s} E_{f} E_{f}^{\mathrm{T}}\left(H_{i}^{s}\right)^{\mathrm{T}}
\end{gathered}
$$

Notice that

$\lim _{j \rightarrow \infty} \frac{1}{j} E_{f} E_{f}^{\mathrm{T}}=\left(\begin{array}{cccc}\Sigma_{a} & & & \\ & \Sigma_{a} & & \\ & & \ddots & \\ & & & \Sigma_{a}\end{array}\right)$

and substituting Eqs. (32) and (26) into (31) yields

$$
\begin{aligned}
\lim _{j \rightarrow \infty} \frac{1}{j}\left(Y_{f}-\Gamma_{i} X_{f}\right)\left(Y_{f}-\Gamma_{i} X_{f}\right)^{\mathrm{T}} & \\
= & \left(\begin{array}{cccc}
\Sigma_{a} & \Sigma_{a} K^{\mathrm{T}} C^{\mathrm{T}} & \cdots & \Sigma_{a} K^{\mathrm{T}}\left(A^{i-2}\right)^{\mathrm{T}} C^{\mathrm{T}} \\
C K \Sigma_{a} & C K \Sigma_{a} K^{\mathrm{T}} C^{\mathrm{T}}+\Sigma_{a} & \cdots & \cdots \\
\cdots & \cdots & \cdots & \cdots \\
C A^{i-2} K & \cdots & \cdots & \cdots A^{i-2} K \Sigma_{a} K^{\mathrm{T}}\left(A^{i-2}\right)^{\mathrm{T}} C^{\mathrm{T}}+\cdots+\Sigma_{a}
\end{array}\right)
\end{aligned}
$$

Comparing Eq. (33) with (22), it is easily established that diagonal elements of $\lim _{j \rightarrow \infty} \frac{1}{j}\left(Y_{f}-\Gamma_{i} X_{f}\right)\left(Y_{f}-\Gamma_{i} X_{f}\right)^{\mathrm{T}}$ are given by

$$
\begin{aligned}
& \operatorname{diag}\left\{\lim _{j \rightarrow \infty} \frac{1}{j}\left(Y_{f}-\Gamma_{i} X_{f}\right)\left(Y_{f}-\Gamma_{i} X_{f}\right)^{\mathrm{T}}\right\} \\
& =\left(\begin{array}{llll}
F_{0} \Sigma_{a} F_{0}^{\mathrm{T}} & & & \\
& F_{1} \Sigma_{a} F_{1}^{\mathrm{T}}+F_{0} \Sigma_{a} F_{0}^{\mathrm{T}} & \\
& & \ddots & \\
& & & F_{i-1} \Sigma_{a} F_{i-1}^{\mathrm{T}}+\cdots+F_{1} \Sigma_{a} F_{1}^{\mathrm{T}}+F_{0} \Sigma_{a} F_{0}^{\mathrm{T}}
\end{array}\right)
\end{aligned}
$$

That is to say, the diagonal elements that are the variance of prediction errors from one step prediction all way through $i$ step prediction, can be calculated easily. Thus, Eq. (34) is a very useful integrated formula that simultaneously calculates the prediction errors over consecutive steps with a single shot of computation. The simultaneous calculation of multiple step prediction errors provides a means to the visualization of the prediction errors $s_{i}$ and closed-loop potentials $p_{i}$ over the extended horizon when the interactor order is unknown.

Next we need to derive $\Gamma_{i} X_{f}$ from data. Considering the fact that the future disturbance $E_{f}$ (white noise) is independent of the past output $Y_{p}$, performing an orthogonal projection of row space of Eq. (27) onto row space of $Y_{p}$ yields $Y_{f} / Y_{p}=\Gamma_{i} X_{f} / Y_{p}$

$X_{f} / Y_{p}$ will be shown in Appendix A to be one realization of the Kalman filter state for the system represented by Eqs. (20) and (21). Therefore, we may write Eq. (35) as

$Y_{f} / Y_{p}=\Gamma_{i} \widehat{X}_{f}$

and $Y_{f} / Y_{p}$ is one estimate of $\Gamma_{i} X_{f}$.

The remaining problem is to calculate the orthogonal (linear) projection used in Eq. (35), $Y_{f} / Y_{p}$, which can be expressed as

$Y_{f} / Y_{p}=L Y_{p}$

The calculation of this projection without relying on the models such as Eqs. (20) and (21) can be formally stated as follows:

Algorithm 1. Given the measurement $Y_{0}, Y_{1}, \ldots, Y_{N}$ of the closed-loop process, calculate prediction errors over a finite horizon using only the output data.

In view of structure of the data Hankel matrices $Y_{p}$ and $Y_{f}$, this problem can be re-casted as: Given $Y_{p}$, find an optimal (in the sense of Frobenius norm) linear predictor of $Y_{f}$ in the form

$\widehat{Y}_{f}=L Y_{p}$

to minimize the Frobenius norm of the prediction error. The problem may be solved by minimization of the following objective

$\min _{L}=\left\|Y_{f}-L Y_{p}\right\|_{F}^{2}$

where the subscript $F$ stands for the Frobenius norm. The solution is well known in subspace literature [27], which is given by the orthogonal projection of the row space of $Y_{f}$ onto the row space of $Y_{p}$, i.e.

$\widehat{Y}_{f}=Y_{f} / Y_{p}=Y_{f} Y_{p}^{+} Y_{p}=Y_{f} Y_{p}^{\mathrm{T}}\left(Y_{p} Y_{p}^{\mathrm{T}}\right)^{-1} Y_{p}$

Therefore,

$L=Y_{f} Y_{p}^{\mathrm{T}}\left(Y_{p} Y_{p}^{\mathrm{T}}\right)^{-1}$

Thus, the optimal predictions over multiple steps can be calculated from the time series data $Y_{t}$ without relaying on an explicit time series model.

Remark 8. Eq. (40) is a regression equation that is known as projection in subspace system identification literature. However, for system identification or time series modeling, one has to extract the system matrices/parameters from the calculated $L$ matrix, i.e. another regression is needed in 
order to estimate model parameters. Therefore, in subspace literature, the projection matrix $L$ has not been treated as a model. However, to obtain an appropriate estimation of $L$, the row dimension of $Y_{f}$ or $Y_{p}$ should be larger than the order of the underlining system as suggested in [27]. Although there is no limitation on how large this row dimension can choose and thus there is some freedom to choose it, a too large row dimension will reduce the column dimension of $Y_{f}$ or $Y_{p}$ and obviously lead to larger variance of estimation of $L$. Since Eq. (40) is essentially a linear least squares problem, the adequacy of the estimation of $L$ can be tested following the standard statistical tests for linear least squares solution from Statistics literature.

On the other hand, if the auto-covariance and covariance of the output data are available, one can also calculate the projection using these covariances matrices. The problem is stated as follows:

Algorithm 2. Given the measurement $Y_{0}, Y_{1}, \ldots, Y_{N}$ of the closed-loop process, calculate prediction errors over a finite horizon using only variance and covariance of the output data.

Let us first define two covariance matrices

$$
\begin{aligned}
\Sigma_{p, p} & =\left(\begin{array}{cccc}
\Lambda_{0} & \Lambda_{-1} & \cdots & \Lambda_{1-i} \\
\Lambda_{1} & \Lambda_{0} & \cdots & \Lambda_{2-i} \\
\cdots & \cdots & \cdots & \cdots \\
\Lambda_{i-1} & \Lambda_{i-2} & \cdots & \Lambda_{0}
\end{array}\right) \\
\Sigma_{f, p} & =\left(\begin{array}{cccc}
\Lambda_{i} & \Lambda_{i-1} & \cdots & \Lambda_{1} \\
\Lambda_{i+1} & \Lambda_{i} & \cdots & \Lambda_{2} \\
\cdots & \cdots & \cdots & \cdots \\
\Lambda_{2 i-1} & \Lambda_{2 i-2} & \cdots & \Lambda_{i}
\end{array}\right)
\end{aligned}
$$

where

$\Lambda_{i}=E\left[Y_{t+i} Y_{t}^{\mathrm{T}}\right]$

It can be verified by direct substitution that

$\lim _{j \rightarrow \infty} \frac{1}{j}\left[Y_{p} Y_{p}^{\mathrm{T}}\right]=\Sigma_{p, p}$

$\lim _{j \rightarrow \infty} \frac{1}{j}\left[Y_{f} Y_{p}^{\mathrm{T}}\right]=\Sigma_{f, p}$

Now, rewrite Eq. (40) as

$L=\frac{1}{j} Y_{f} Y_{p}^{\mathrm{T}}\left(\frac{1}{j} Y_{p} Y_{p}^{\mathrm{T}}\right)^{-1}$

As $j \rightarrow \infty$

$L \rightarrow \Sigma_{f, p}\left(\Sigma_{p, p}\right)^{-1}$

Thus the optimal prediction over multiple steps is given by

$\widehat{Y}_{f}=\Sigma_{f, p}\left(\Sigma_{p, p}\right)^{-1} Y_{p}$

where $\Sigma_{f, p}$ and $\Sigma_{p, p}$ can be easily calculated from variance and covariance of time series data $Y_{t}$. No time series modeling is needed.

To illustrate the proposed algorithms, re-consider Example 7. With $k=3$, a set of 2000 data points is simulated. Optimal prediction errors $s_{i}$ and closed-loop potentials $p_{i}$ are estimated from simulated routine operating data and compared to their theoretical values in Fig. 5. One can see that the estimated results have a good agreement with the theoretical ones.

Remark 9. To use the proposed algorithm, one should have one set of routine operating data for one controller
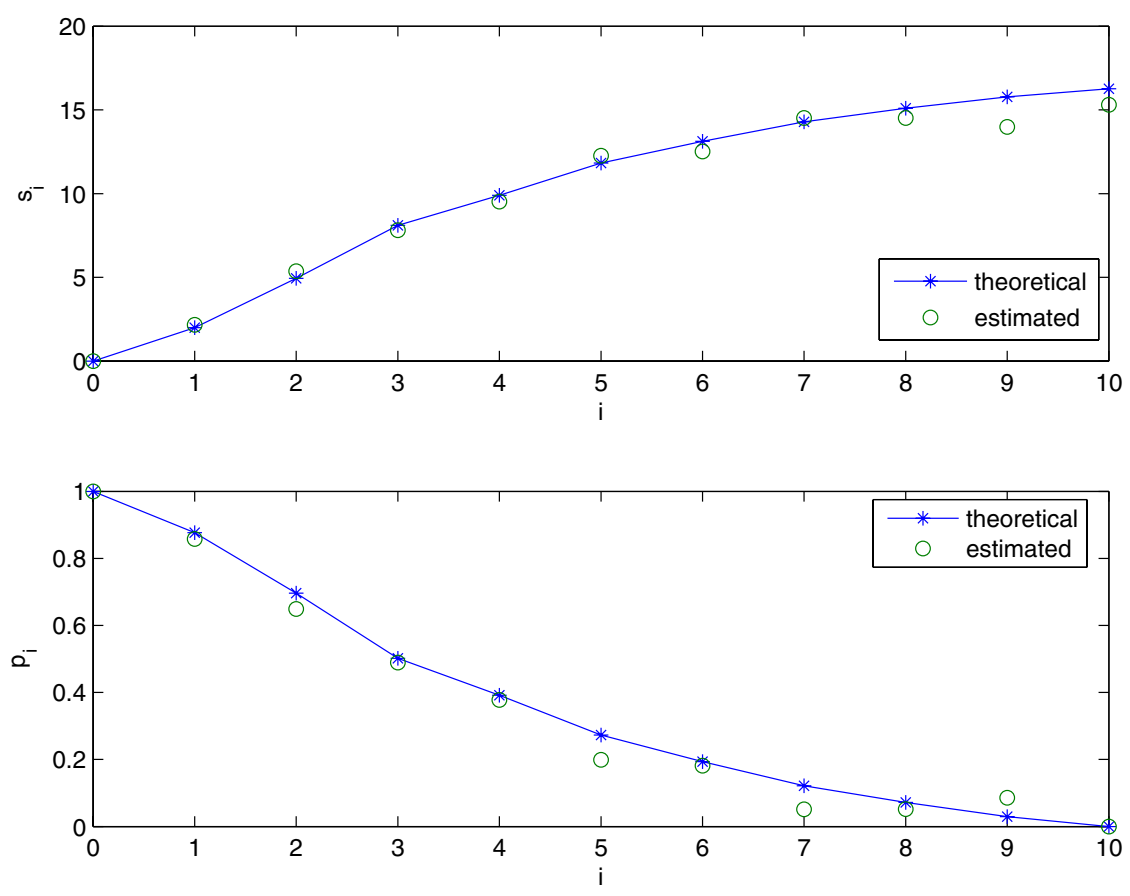

Fig. 5. Theoretical and estimated $s_{i}$ and $r_{i}$ plots. 
setting and then calculate one $p$ versus $i$ plot, which is used as a reference. A natural question is how this set of data can be obtained and the proposed algorithm can be applied. There are several practical scenarios in which the proposed algorithms can be applied. (1) When a control engineer is setting up a new multi-variable control structure there would be opportunity to experiment with different tunings and build up a reference family of curves that can be compared with the potential curves in future. (2) Process control engineers could have a general engineering judgement for how their process should behave. This is similar to the user defined benchmark $[19,12]$. They would often know that it should be settled after a certain elapsed time and therefore be able to make a user-defined potential curve that is a "good enough" target for the multi-variable controller. (3) When control engineers perform field tuning/re-tuning of the controllers, this algorithm provides the direction of the tunings without involving complex calculations. One caution, when apply this method or even other control performance methods, is that the dynamics and covariance structure of the disturbance should remain the same throughout performance assessment process to ensure a fair comparison of control performance before and after the tuning.

\subsection{Case study}

For a petrochemical distillation column, shown in Fig. 6, which separates chemical petro in a refinery of Shell Company in Cologne (FRG) in Germany, a multi-variable predictive controller has been developed [28]. Its feed is chemical petrol from the desulfurization, its top product is light petro with boiling point between 30 and $65^{\circ} \mathrm{C}$, and its bottom product is heavy petro with boiling point between 65 and $180^{\circ} \mathrm{C}$.

According to the multi-variable model provided in [28], we have designed an MPC using MATLAB MPC toolbox. In this section, we will use the proposed data-driven algo-

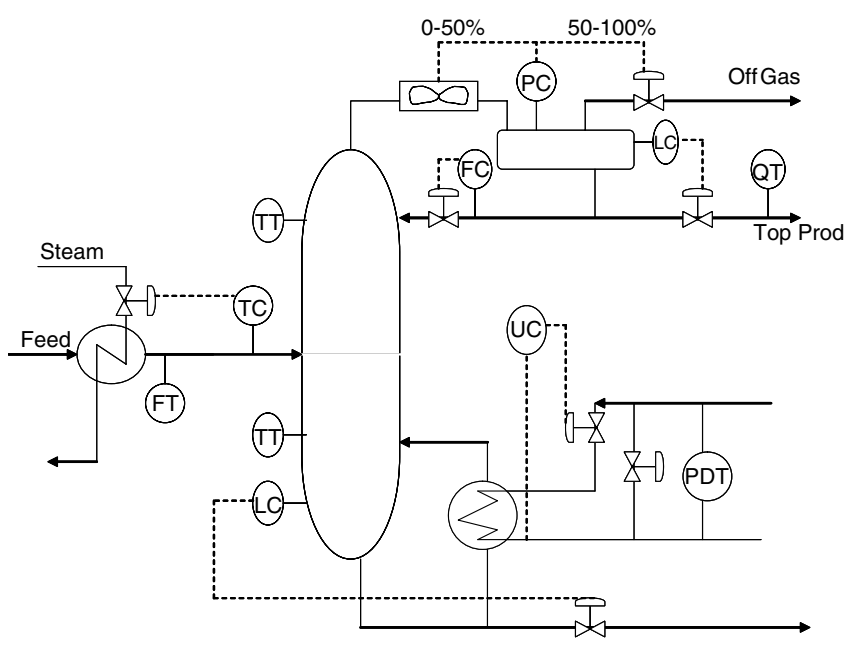

Fig. 6. Schematic diagram of distillation column. rithms to evaluate closed-loop potentials for various tunings.

This process has 10 controlled variables $(\mathrm{CVs})$, four manipulated variables (MVs) and one disturbance variable, plus noise in most of CVs. All CVs/MVs and their corresponding parameters are shown in Tables 3 and 4.

According to [28], the first three CVs are quality variables. The concentration of the heavy petro in the top product (distillate) has to be kept below $2.5 \%$. The concentration is estimated by the final boiling point (FBP), i.e. the boiling temperature of the product if $99 \%$ of the product has been evaporated. For bottom product quality, the pressure compensated temperature (PCT) is calculated and used as CV. Meanwhile, the column pressure has to be kept at its minimum value in order to reduce the energy consumption (reboiler heating). CV4 and CV5 are auxiliary variables that are controlled with less accuracy than the first three main controlled variables. The remaining $\mathrm{CVs}$ are just constrained variables.

To reflect model-plant mismatch, rather than using the exact model for the MPC design, an approximate model through 'linearization' function in Simulink by perturbing the original model is obtained and the MPC controller is designed based on the approximate model. The designed MPC controller is then implemented in the original model. All CVs except for CV6 and CV8 are subject to disturbance, which is filtered white noise with filter time constant 5 min. CV6 and CV8 (OPs) can be measured exactly and are not subject to the noise. A disturbance variable (DV) is the feed flow rate, which is simulated by filtered white noise with filter time constant $10 \mathrm{~min}$.

Table 3

MVs and their constraints, weights used in MPC design

\begin{tabular}{llllll}
\hline No. & MV & Min & Max & Rate & Weight \\
\hline 1 & $\begin{array}{l}\text { Reflux PID } \\
\text { setpoint }\end{array}$ & $299 \mathrm{t} / \mathrm{d}$ & $700 \mathrm{t} / \mathrm{d}$ & $20 \mathrm{t} / \mathrm{d}$ & 0.0059 \\
2 & $\begin{array}{l}\text { Pressure PID } \\
\text { setpoint }\end{array}$ & $0.145 \mathrm{bar}$ & $0.6 \mathrm{bar}$ & $0.001 \mathrm{bar} / \mathrm{min}$ & $1,000,000$ \\
3 & $\begin{array}{l}\text { Feed } \\
\text { temperature }\end{array}$ & $0 \%$ & $100 \%$ & $0.5 \% / \mathrm{min}$ & 0.5153 \\
4 & Duty valve & $62 \%$ & $100 \%$ & $0.1 \% / \mathrm{min}$ & 1.7374 \\
\hline
\end{tabular}

Table 4

CVs and their constraints, weights used in MPC design

\begin{tabular}{llllll}
\hline No. & CV & Unit & Min & Max & Weight \\
\hline 1 & Final boil point (top) & ${ }^{\circ} \mathrm{C}$ & 67.75 & 68.25 & 1 \\
2 & $\begin{array}{l}\text { Pressure corrected } \\
\text { temperature (bottom) }\end{array}$ & ${ }^{\circ} \mathrm{C}$ & 85.5 & 86.5 & 0.04 \\
3 & Pressure & bar & 0.15 & 0.15 & 3.33 \\
4 & Feed temperature & ${ }^{\circ} \mathrm{C}$ & 70.0 & 70.0 & 0.01 \\
5 & Pressure corrected & ${ }^{\circ} \mathrm{C}$ & 56.0 & 66.0 & 1.0 \\
& temperature (top) & & & & \\
6 & Pressure PID valve position & $\%$ & 0 & 42 & 25 \\
7 & Duty & $\mathrm{MW}$ & 4.0 & 5.5 & 1.0 \\
8 & Baypass valve position & $\%$ & 0 & 95 & 1.0 \\
9 & Reflux PID flow & $\mathrm{t} / \mathrm{d}$ & 0 & 650 & 0.0001 \\
10 & Furnace duty violation & $\mathrm{MW}$ & -1000 & 0 & 1.25 \\
\hline
\end{tabular}


For the MPC design, sampling time is chosen as $1 \mathrm{~min}$, prediction horizon as 10 samples, and control horizon as two samples. Since CV3 and CV4 are setpoint tracking variables (same upper and lower constraints), step disturbance is selected in the MPC design GUI in MPC toolbox. We consider different tunings by adjusting "Overall" performance knob from 0 (Most robust), 0.5 (Median), to 1 (Fastest response). These three tunings are labelled as Tuning 1,2 and 3. Tuning 4 has the same parameters as Tuning 2 except for the weighting of CV1 being increased from 1 to 100 .

In computing overall closed-loop potential, we have considered the weighting of each CV. The data of each $\mathrm{CV}$ are scaled by the square root of corresponding weighting of that $\mathrm{CV}$ before performing any further computations. Comparison of overall closed-loop potentials for Tuning 1, 2 and 3 is shown in Fig. 7. The single numerical measures of the three relative potentials are -0.048 , and -0.054 with Tuning 2 as reference, respectively. These numerical measures show that there is not much change in the performance among the first three tunings. However, by visualizing individual closed-loop potentials shown in Figs. $8-10$, one can see that there is some difference between Tuning 1 and Tuning 2/3, while Tuning 2 and 3 are quite similar. The difference lies in the FBP (CV1) which is the most important quality variable. Both tuning 2 and 3 deteriorate the control of this critical CV by slightly improving other two $\mathrm{CV}$, relative to Tuning 1 . Therefore, Tuning 1 is recommended among these three tunings.

The individual closed-loop potentials also indicate that the first three tunings do not yield good control performance for FBP compared to other two critical CVs, that is, it has slowest decaying closed-loop potential in all three tunings. Considering that the improvement of the control of FBP is the most important, we simulate Tuning 4 (with more weight on FBP) and the comparison of overall closed-loop potentials for all four tunings is shown in Fig. 12. The numerical closed-loop potential index for

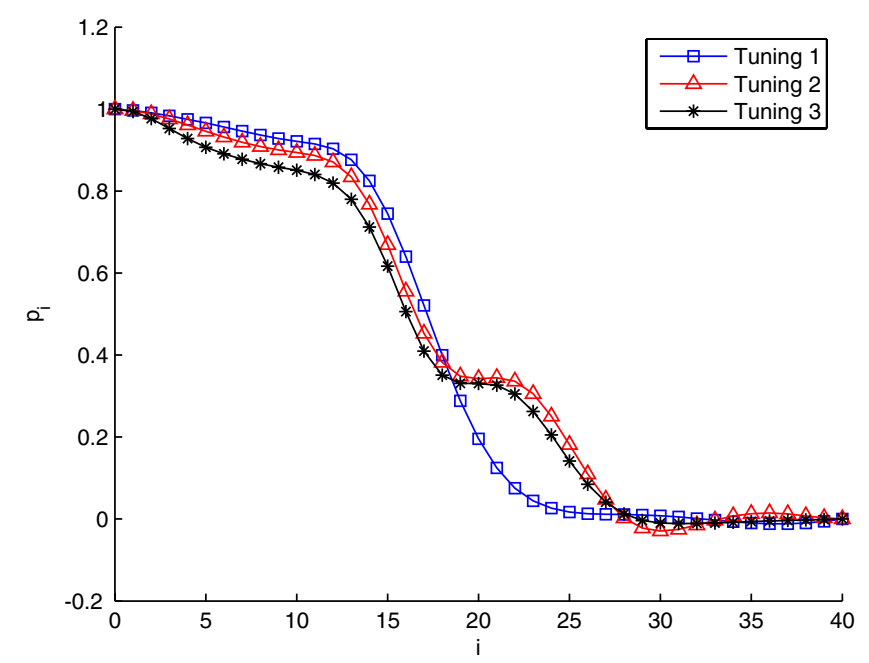

Fig. 7. Comparison of overall closed-loop potentials from Tuning 1, 2 and 3 for three quality CVs.

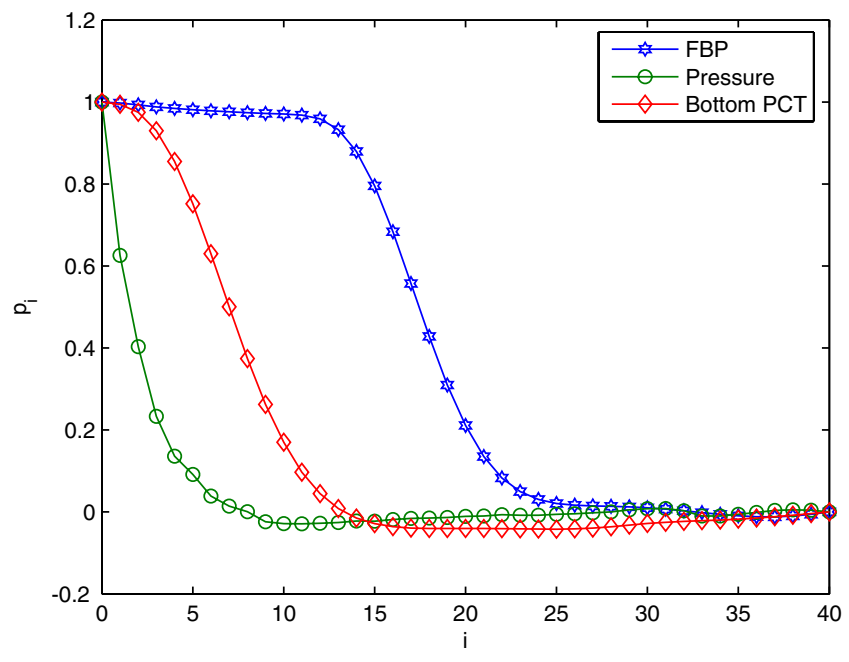

Fig. 8. Individual closed-loop potentials from Tuning 1 for three quality CVs.

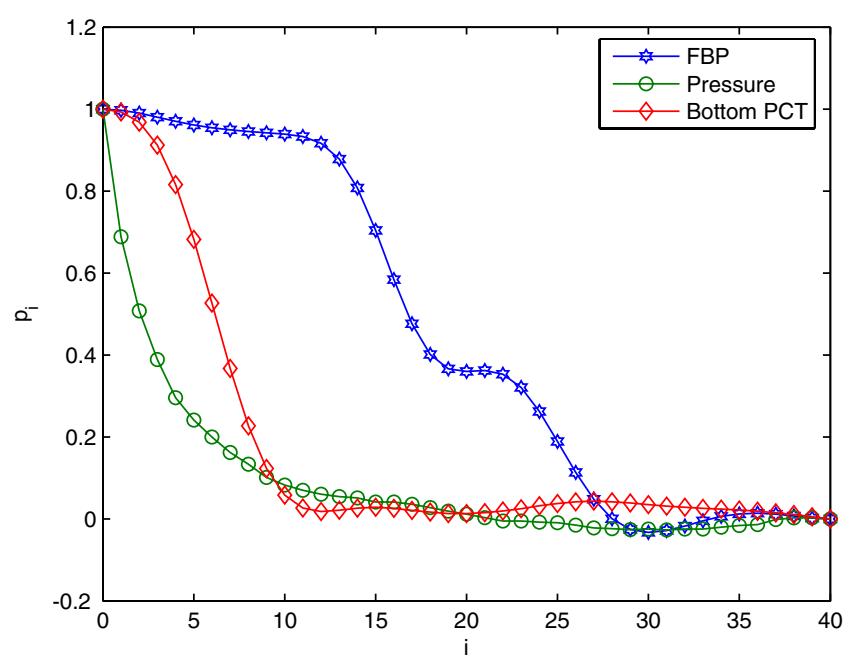

Fig. 9. Individual closed-loop potentials from Tuning 2 for three quality CVs.

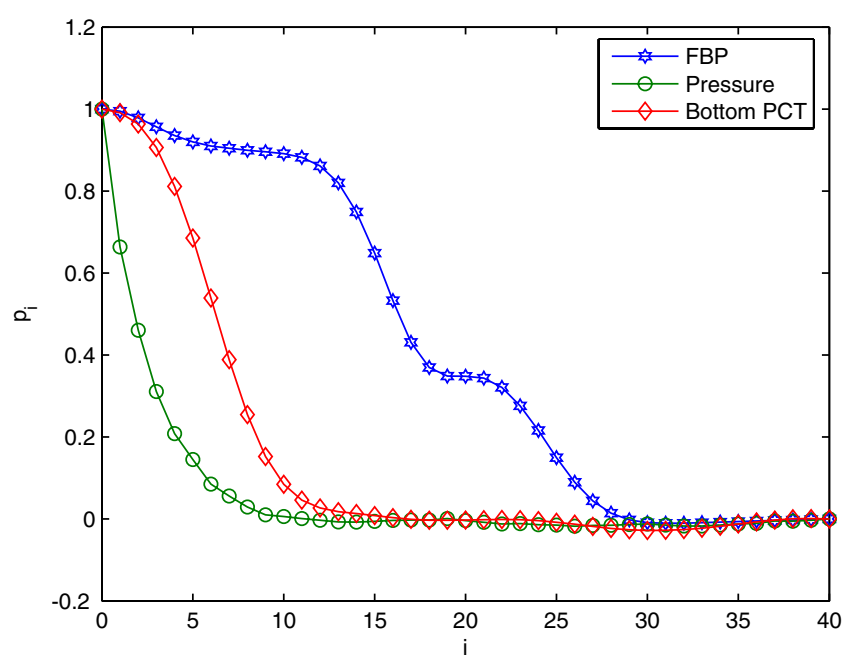

Fig. 10. Individual closed-loop potentials from Tuning 3 for three quality CVs. 


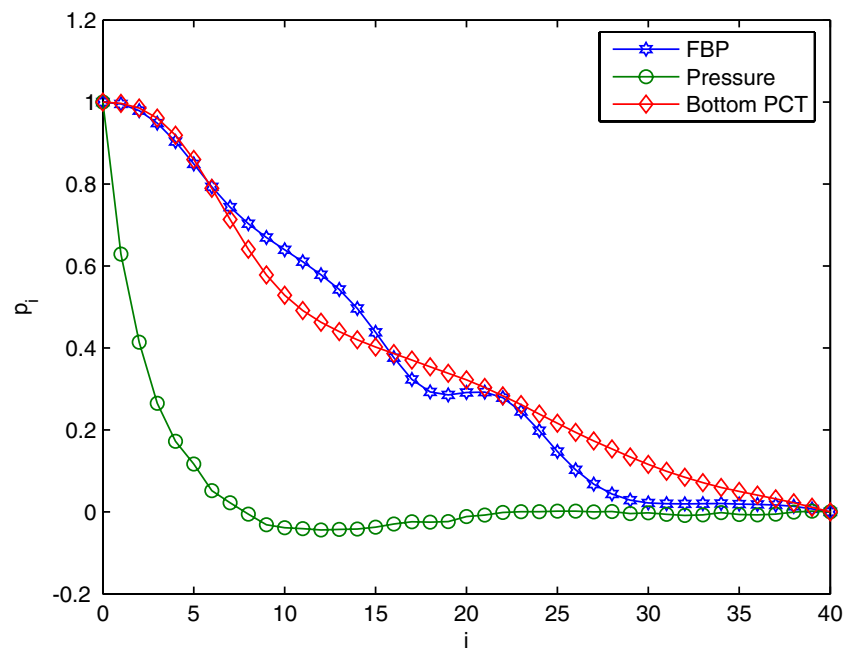

Fig. 11. Individual closed-loop potentials from Tuning 4 for three quality CVs.

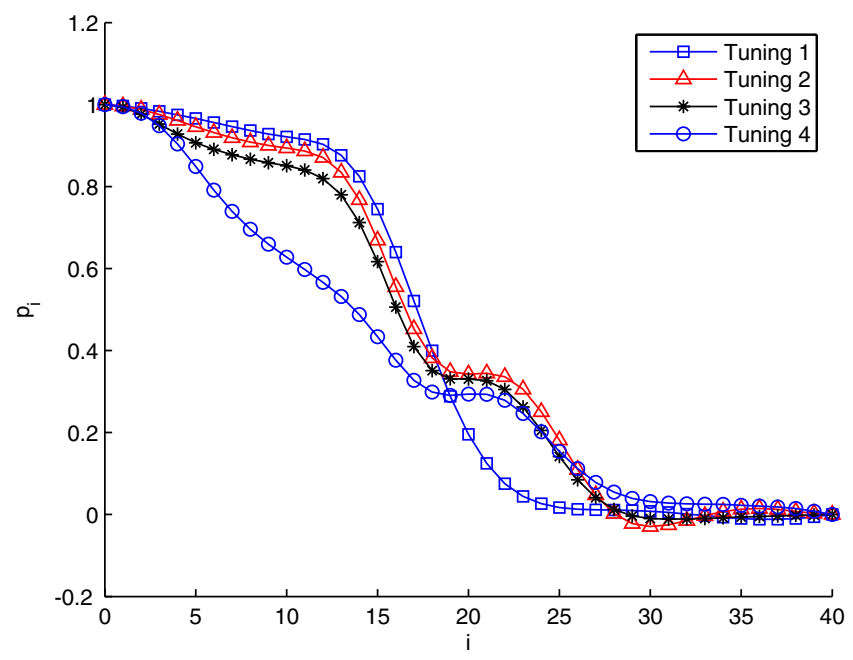

Fig. 12. Comparison of overall closed-loop potentials from Tuning 1, 2, 3 and 4 for three quality CVs.

Tuning 4 is -0.17 with Tuning 2 as the reference, a clear indication of improved performance. It therefore turns out that Tuning 4 is the best among the four tunings in terms of the overall performance. The individual closedloop potential shown in Fig. 11 indicates the improvement comes from the improvement of FBP at the cost of Bottom PCT, showing a tradeoff between top product quality and bottom product quality.

\section{Conclusion}

In this paper, we have discussed alternative and simple solutions to multi-variate feedback control performance assessment. We have considered two scenarios: known pair-wise time delays and no a priori knowledge of the interactor matrices. For the case of known pair-wise time delays, we have discussed procedure for determining and extracting diagonal interactor matrices. For the case of no a priori knowledge, we have proposed a performance measure based on closed-loop potential. The solution is based on the multi-step optimal prediction error. Two data-driven subspace algorithms have been developed to compute the optimal prediction errors and closed-loop potentials. The simulation examples and a distillation column case study have shown the features of the proposed algorithms.

\section{Acknowledgements}

This work is supported in part by Humboldt Research Fellowship of Germany and Natural Sciences and Engineering Research Council of Canada.

\section{Appendix A. Non-steady state Kalman filters}

A non-steady state Kalman filter can be derived from Eqs. (27)-(29) using the following procedure:

First, from Eq. (28), one can obtain

$E_{p}=H_{i}^{-s} Y_{p}-H_{i}^{-s} \Gamma_{i} X_{p}$

Substituting Eq. (A.1) into Eq. (29) yields

$X_{f}=\left(A^{i}-\Delta_{i}^{s} H_{i}^{-s} \Gamma_{i}\right) X_{p}+\Delta_{i}^{s} H_{i}^{-s} Y_{p}$

It is easy to see that $X_{f}$ is the non-steady state solution of Kalman filter state with $X_{p}$ as its initial value since it is derived from Eqs. (27)-(29), which, in turn, are derived from the innovation form of the state space model, Eqs. (20) and (21). The innovation form is known as the Kalman filter $[5,20]$.

In subspace literature, one often performs an oblique projection [27] of Eq. (A.2) on to $W_{p}$ through $R$ where $R$ is any constant matrix of appropriate dimension

$$
\begin{aligned}
X_{f} /{ }_{R} W_{p}= & \left(A^{i}-\Delta_{i}^{s} H_{i}^{-s} \Gamma_{i}\right) X_{p} /{ }_{R} W_{p}+\left(\Delta_{i}^{s} H_{i}^{-s} \mid \Delta_{i}^{d}\right. \\
& \left.-\Delta_{i}^{s} H_{i}^{-s} H_{i}^{d}\right) W_{p} /{ }_{R} W_{p}
\end{aligned}
$$

$W_{p} /{ }_{R} W_{p}=W_{p}$ by the definition of oblique projection. Therefore, Eq. (A.3) can be simplified to

$$
\begin{aligned}
X_{f} /{ }_{R} W_{p}= & \left(A^{i}-\Delta_{i}^{s} H_{i}^{-s} \Gamma_{i}\right) X_{p} /{ }_{R} W_{p}+\left(\Delta_{i}^{s} H_{i}^{-s} \mid \Delta_{i}^{d}\right. \\
& \left.-\Delta_{i}^{s} H_{i}^{-s} H_{i}^{d}\right) W_{p}
\end{aligned}
$$

Comparing Eq. (A.4) with (A.2), one can see that these two equations have the exactly the same structure, as the solution of the innovation state space equations (20) and (21). In Eq. (A.2), $X_{f}$ is the Kalman filter state with $X_{p}$ as its initial condition. In Eq. (A.4), $X_{f} /_{R} W_{p}=\widehat{X}_{f}$ is Kalman filter state but with the initial condition $X_{p} /{ }_{R} W_{p}=\widehat{X}_{p}$.

Following the same line, if we now make a orthogonal projection of Eq. (A.2) onto $Y_{p}$, we should have

$X_{f} / Y_{p}=\left(A^{i}-\Delta_{i}^{s} H_{i}^{-s} \Gamma_{i}\right) X_{p} / Y_{p}+\Delta_{i}^{s} H_{i}^{-s} Y_{p}$

and we can equally treat $X_{f} / Y_{p}$ as another Kalman filter state but with $X_{p} / Y_{p}$ as its initial condition, i.e.

$\widehat{X}_{f}=X_{f} / Y_{p}$ 
with initial state as

$\widehat{X}_{p}=X_{p} / Y_{p}$

\section{References}

[1] K.J. Astrom, Introduction to Stochastic Control Theory, Academic Press, New York, 1970.

[2] W.R. DeVries, S.M. Wu, Evaluation of process control effectiveness and diagnosis of variation in paper basis weight via multivariate time series analysis, IEEE Trans. AC AC-23 (4) (1978).

[3] L. Dugard, G.C. Goodwin, X. Xianya, The role of the interactor matrix in multivariable stochastic adaptive control, Automatica 20 (5) (1984) 701-709.

[4] J. Gao, R. Patwardhan, K. Akamatsu, Y. Hashimoto, G. Emonto, S.L. Shah, B. Huang, Performance evaluation of two industrial MPC controllers, Control Eng. Pract. 11 (2003) 1371-1387.

[5] G.C. Goodwin, K.S. Sin, Adaptive Filtering Prediction and Control, Prentice-Hall, Englewood Cliffs, 1984.

[6] T. Harris, Assessment of closed loop performance, Can. J. Chem. Eng. 67 (1989) 856-861.

[7] T.J. Harris, F. Boudreau, J.F. MacGregor, Performance assessment of multivariable feedback controllers, Automatica 32 (11) (1996) 1505-1518.

[8] T.J. Harris, C.T. Seppala, L.D. Desborough, A review of performance monitoring and assessment techniques for univariate and multivariate control systems, J. Process Control 9 (1999) 1-17.

[9] A. Horch, G. Dumont, Control loop performance assessment (Guest editorial), Int. J. Adapt. Control Signal Process. 17 (2003) 523-525 (special issue).

[10] B. Huang, S.X. Ding, Assessing limit of multivariable feedback control performance with reduced prior requirement on process knowledge, in: Controlo'04, Faro, Portugal, 2004.

[11] B. Huang, S.X. Ding, N. Thornhill, Practical solutions to multivariate feedback control performance assessment problem: reduced a priori knowledge of interactor matrix, J. Process Control 15 (2005) 573-583.

[12] B. Huang, S.L. Shah, Control Loop Performance Assessment: Theory and Applications, Springer Verlag, London, 1999.

[13] B. Huang, S.L. Shah, K.Y. Kwok, How good is your controller? Application of control loop performance assessment techniques to MIMO processes, in: Proceedings of the 13th IFAC World Congress, San Francisco, vol. M, July 1996, pp. 229-234.
[14] B. Huang, S.L. Shah, K.Y. Kwok, Good, bad or optimal? Performance assessment of MIMO processes, Automatica 33 (6) (1997) 1175-1183.

[15] H.P. Huang, J.C. Jeng, C.H. Chiang, W. Pan, A direct method for multi-loop PI/PID controller design, J. Process Control 13 (2003) 769-786.

[16] R. Kadali, B. Huang, Multivariate control performance assessment without interactor matrix, in: IFAC Advanced Control of Chemical Processes, 2003, pp. 61-66.

[17] R. Kadali, B. Huang, E.C. Tamayo, A case study on performance analysis and troubling shooting of an industrial model predictive control system, in: Proceedings of 1999 American Control Conference, 1998, pp. 642-646.

[18] B.S. Ko, T.F. Edgar, Performance assessment of multivariate feedback control systems, Automatica 37 (5) (2001) 899-905.

[19] D.J. Kozub, Controller performance monitoring and diagnosis: experience and challenges, AIChE Symp. Ser. 93 (316) (1997) 83.

[20] L. Ljung, System Identification, second ed., Prentice-Hall, 1999.

[21] C.A. McNabb, S.J. Qin, Projection based MIMO control performance monitoring: I-covariance monitoring in state space, J. Process Control 13 (2003) 739-757.

[22] Y. Mutoh, R. Ortega, Interactor structure estimation for adaptive control of discrete-time multivariable nondecouplable systems, Automatica 29 (3) (1993) 635-647.

[23] C.T. Seppala, T.J. Harris, D.W. Bacon, Time series methods for dynamic analysis of multiple controlled variables, J. Process Control 12 (2002) 257-276.

[24] S.L. Shah, C. Mohtadi, D.W. Clarke, Multivariable adaptive control without a prior knowledge of the delay matrix, Syst. Control Lett. 9 (1987) 295-306

[25] S.L. Shah, R. Patwardhan, B. Huang, Multivariate controller performance assessment: methods, applications and challenges, in: Chemical Process Control-CPC VI, CACHE, Tuscon, AZ, 2002, pp. 190-207.

[26] N.F. Thornhill, M. Oettinger, P. Fedenczuk, Refinery-wide control loop performance assessment, J. Process Control 9 (2) (1999) 109124.

[27] P. van Overschee, B.D. Moor, Subspace Identification for Linear Systems, Kluwer Academic Publishers, Boston, 1996.

[28] U. Volk, D.W. Kniese, R. Hahn, R. Haber, U. Schmitz, Optimized multivariable predictive control of an industrial distillation column considering hard and soft constraints, Control Eng. Pract. 13 (2005) 913-927. 